\title{
Is it time to reframe how we care for people with non-traumatic musculoskeletal pain?
}

\author{
Jeremy Lewis, ${ }^{1,2}$ Peter O'Sullivan ${ }^{3,4}$
}

\section{THE CURRENT APPROACH TO MUSCULOSKELETAL PAIN IS FAILING}

The majority of persistent non-traumatic musculoskeletal pain disorders do not have a pathoanatomical diagnosis that adequately explains the individual's pain experience and disability. We contend this has resulted in two concerning developments in the management of people with such disorders. First, structural changes observed on imaging that are highly prevalent in pain free populations, such as rotator cuff tears, intervertebral disc degeneration, labral tears and cartilage changes, are ascribed to individuals as a diagnosis for their condition. In this context, this information may result in the individual believing that their body is damaged, fragile and in need of protection, resulting in a cascade of movement and activity avoidance behaviours and seeking interventions to correct the structural deficits. ${ }^{1}$ This trend has led to exponential increases in elective surgery rates and associated costs, while the efficacy of repairing (eg, rotator cuff and medical meniscal tears), reshaping (eg, subacromial decompression) or replacing (eg, lumbar intervertebral discs) the structures considered to be at fault has been substantially challenged. ${ }^{2-10}$ Second, it is arguable that musculoskeletal clinicians have invented treatments for conditions that may not exist or be readily detected (such as trigger points, sacral torsions), and they have developed and perpetuated treatment paradigms (such as 'correcting' upper body posture and muscle imbalances) that do not conform to current research evidence. ${ }^{11-14}$ These two trends have created an expectation that interventions (frequently 'passive') will provide a

\footnotetext{
${ }^{1}$ School of Health and Social Work, University of Hertfordshire, Hertfordshire, UK

${ }^{2}$ Therapy Department, Central London Community Healthcare National Health Service Trust, London, UK ${ }^{3}$ Health Sciences Division, School of Physiotherapy and Exercise Science, Curtin University, Perth, Western Australia, Australia

${ }^{4}$ Bodylogic Physiotherapy, Perth, Western Australia, Australia
}

Correspondence to Professor Jeremy Lewis, School of Health and Social Work, University of Hertfordshire, Hertfordshire AL10 9AB, UK;

jeremy.lewis@LondonShoulderClinic.com 'cure', and typically quickly, with minimal self-contribution. This expectation may have been derived from a conversation with a friend or family member, from the Internet or from an advertising campaign, but almost certainly originated from health professionals.

\section{WHAT CAN WE LEARN FROM THE MANAGEMENT OF OTHER CHRONIC HEALTH CONDITIONS?}

Contemporary evidence demonstrates that many musculoskeletal pain conditions are associated with long term disability that are often resistant to current treatments. They are influenced by multiple interacting factors, including genetics, psychological, social and biophysical factors, comorbidities and lifestyle. ${ }^{15}$ We propose that when these conditions become persistent and disabling they should be viewed in a similar way to other chronic health conditions. For example, when an individual presents with non-insulin dependent diabetes, best practice would involve an interview and examination where a diagnosis is reached and the relevant biopsychosocial contributing factors to the disorder are identified. Based on this, education, advice and shared decision making, a management plan, underpinned with empathy and support, would be agreed. This plan would typically involve highlighting the importance of good sleep hygiene, the role of nutrition and diet, stress management and, if relevant, cessation or reduction of smoking. The profound benefits of participating in appropriate graduated physical activity would be emphasised and, if necessary, medication, such as metformin, may also be prescribed. It also involves the management of comorbid health complaints. While, if required, the prescribed medicine would differ, the management of other chronic disorders, such as asthma and high blood pressure, would follow a similar process.

In the management of these conditions, the focus is not on providing a 'cure' but rather the discussion is about providing a 'management' plan to control the disorder and limit its impact on the person's well-being. While the signs and symptoms of many of these chronic problems may reduce to the level that the individual no longer feels disabled or symptomatic, ongoing self-management is essential.

\section{A NEW APPROACH IS NEEDED}

We believe there is a need to reframe the way we care for non-traumatic persistent and disabling musculoskeletal pain conditions, by aligning the management of such conditions with the principles underpinning the management of other chronic conditions: strong clinical alliance, education, exercise and lifestyle (sleep hygiene, smoking cessation, stress management, etc) in order to build the individual's self-efficacy to take control and ultimately be responsible for their health. Although the arguments for such an approach are compelling, ${ }^{16}$ and evidence is emerging for a number of musculoskeletal disorders, it is acknowledged that definitive evidence for all musculoskeletal conditions is currently lacking. Such an approach would be supported by other and emerging evidence, translated from the available research for the condition being managed.

Reframed in this manner, patients would no longer be led to expect a 'magic' manipulation or other passive approach to 'cure' their condition, and this in turn may reduce stress and burnout experienced by many clinicians who are unable to deliver on such unsubstantiated promises. Interventions such as manual therapy, pharmacology and injections, when provided, should be seen as an adjunct, and their risks and benefits must be considered and honestly communicated.

\section{OBSTACLES AND OPPORTUNITIES FOR THIS APPROACH}

We need to reframe what is currently doable and achievable in the management of many non-traumatic musculoskeletal presentations, and honest and open conversations regarding the outcome evidence for these disorders needs to be sensitively communicated. There would be many obstacles to overcome and respect when considering such an approach. For clinicians, these might be pain beliefs, professional identity, time, financial pressures and lack of adequate training. Patient beliefs and expectations may also pose a significant challenge for clinicians, especially when they desire a structural diagnosis and a 
'fix' for their pain. For patients, creating an understanding and expectation that, as with other chronic health conditions, there is no magic cure for many persistent and disabling musculoskeletal pain conditions, and that ultimately ongoing evidence informed self-management is the key. To achieve this, the efforts of many institutions, including educational, healthcare, political and professional organisations, health funding bodies and the media, need to be involved.

We believe in the need to frame past beliefs against new evidence, and when in conflict we need to evolve with that evidence. We contend this requires those of us working in the musculoskeletal field to acknowledge the limitations of current surgical and non-surgical interventions for persistent and disabling non-traumatic presentations, as well as upskill and reframing of our practice, language and expectations to consider aligning our current practice with that supporting most chronic healthcare conditions. By doing this we can better support those members of our societies who seek care, and be more honest with the level and type of care we can and should currently offer, and the outcomes that may be achieved.

Correction notice This article has been corrected since it was published Online First. Two typographical errors have been corrected.
Contributors Both authors equally contributed to the idea, writing and editing of the editorial.

Funding The authors have not declared a specific grant for this research from any funding agency in the public, commercial or not-for-profit sectors.

Competing interests None declared.

Patient consent Not required.

Provenance and peer review Not commissioned; externally peer reviewed.

(c) Article author(s) (or their employer(s) unless otherwise stated in the text of the article) 2018. All rights reserved. No commercial use is permitted unless otherwise expressly granted.

\section{Check for updates}

To cite Lewis J, O'Sullivan P. Br J Sports Med 2018;52:1543-1544.

Accepted 23 May 2018

Published Online First 25 June 2018

Br J Sports Med 2018;52:1543-1544.

doi:10.1136/bjsports-2018-099198

\section{REFERENCES}

1 Bunzli S, Smith A, Schütze R, et al. Making sense of low back pain and pain-related fear. J Orthop Sports Phys Ther 2017;47:628-36.

2 Beard DJ, Rees JL, Cook JA, et al. Arthroscopic subacromial decompression for subacromial shoulder pain (CSAW): a multicentre, pragmatic, parallel group, placebo-controlled, three-group, randomised surgical trial. Lancet 2018;391:329-38.

3 Moseley JB, O'Malley K, Petersen NJ, et al. A controlled trial of arthroscopic surgery for osteoarthritis of the knee. N Engl J Med 2002;347:81-8.

4 Schrøder CP, Skare Ø, Reikerås 0, et al. Sham surgery versus labral repair or biceps tenodesis for type II SLAP lesions of the shoulder: a three- armed randomised clinical trial. Br J Sports Med 2017:51:1759-66.

5 Sihvonen R, Paavola M, Malmivaara A, et al. Arthroscopic partial meniscectomy versus sham surgery for a degenerative meniscal tear. N Engl I Med 2013:369:2515-24.

6 Hunter DJ. Osteoarthritis management: time to change the deck. J Orthop Sports Phys Ther 2017;47:370-2.

7 Jonas WB, Crawford C, Colloca L, et al. To what extent are surgery and invasive procedures effective beyond a placebo response? A systematic review with metaanalysis of randomised, sham controlled trials. BMJ Open 2015;5:e009655.

8 Harris I. Surgery, the ultimate placebo. Sydney: NewSouth Publishing, 2016.

9 Lewis J. The end of an era? J Orthop Sports Phys Ther 2018;48:127-9.

10 Jacobs WC, van der Gaag NA, Kruyt MC, et al. Total disc replacement for chronic discogenic low back pain: a Cochrane review. Spine 2013:38:24-36.

11 Lucas N, Macaskill P, Irwig L, et al. Reliability of physical examination for diagnosis of myofascial trigger points: a systematic review of the literature. Clin J Pain 2009;25:80-9

12 Cooperstein R, Hickey M. The reliability of palpating the posterior superior iliac spine: a systematic review. J Can Chiropr Assoc 2016:60:36-46.

13 Lewis JS, Green A, Wright C. Subacromial impingement syndrome: the role of posture and muscle imbalance. J Shoulder Elbow Surg 2005;14:385-92.

14 Ratcliffe E, Pickering S, McLean S, et al. Is there a relationship between subacromial impingement syndrome and scapular orientation? A systematic review. Br J Sports Med 2014:48:1251-6.

15 Hartvigsen J, Hancock MJ, Kongsted A, et al. What low back pain is and why we need to pay attention. Lancet 2018.

16 Dean E, Söderlund A. What is the role of lifestyle behaviour change associated with non-communicable disease risk in managing musculoskeletal health conditions with special reference to chronic pain? BMC Musculoskelet Disord 2015;16:87.

\section{GLA:D to have a high-value option for patients with knee and hip arthritis across four continents: Good Life with osteoArthritis from Denmark}

\author{
Ewa M Roos, ${ }^{1}$ Christian J Barton, ${ }^{2,3}$ Aileen M Davis, ${ }^{4,5}$ \\ Rhona McGlasson, ${ }^{6}$ Joanne L Kemp, ${ }^{3}$ Kay M Crossley, ${ }^{3}$ Qiang Liu, \\ Jianhao Lin, ${ }^{7}$ Søren T Skou ${ }^{1,8}$
}

\section{WHAT IS THE PROBLEM?}

We know that we should be providing patient education, exercise and weight management for people with knee and hip osteoarthritis, but do we know what to do? GLA:D may provide an answer,

\footnotetext{
${ }^{1}$ Research Unit for Musculoskeletal Function and Physiotherapy, University of Southern Denmark, Odense, Denmark

${ }^{2}$ University of Melbourne Department of Surgery, St. Vincent's Hospital, Australia

${ }^{3}$ LaTrobe Sport and Exercise Medicine Research Centre, School of Allied Health, College of Science, Health and Engineering, La Trobe University, Melbourne, Australia ${ }^{4}$ Department of Physical Therapy, Institute of Health

Policy, Managementand Evaluation, University of Toronto, Toronto, Canada

${ }^{5}$ Health Care and Outcomes Research, Krembil Research Institute, University Health Network, Toronto, Canada ${ }^{6}$ Bone and Joint Canada, Canadian Orthopaedic Foundation, Toronto, Canada

${ }^{7}$ Arthritis Clinic and Research Center, Peking University People's Hospital, Beijing, China

${ }^{8}$ Department of Physiotherapy and Occupational Therapy, Næstved-Slagelse-Ringsted Hospitals, Slagelse, Denmark
}

Correspondence to Ewa M Roos, University of Southern Denmark, Campusvej 55, DK-5230 Odense M, Denmark; eroos@health.sdu.dk 